\title{
Identification of gene fusion transcripts by transcriptome sequencing in BRCA1-mutated breast cancers and cell lines
}

Kevin CH Ha ${ }^{1,2}$, Emilie Lalonde ${ }^{1,2}$, Lili Li ${ }^{1,3,4}$, Luca Cavallone ${ }^{3,4}$, Rachael Natrajan ${ }^{5}$, Maryou B Lambros ${ }^{5}$, Costas Mitsopoulos ${ }^{5}$, Jarle Hakas ${ }^{5}$, Iwanka Kozarewa ${ }^{5}$, Kerry Fenwick ${ }^{5}$, Chris J Lord ${ }^{5}$, Alan Ashworth ${ }^{5}$, Anne Vincent-Salomon ${ }^{6}$, Mark Basik ${ }^{4,7,8}$, Jorge S Reis-Filho ${ }^{5}$, Jacek Majewski ${ }^{1,2}$ and William D Foulkes ${ }^{1,3,4,7^{*}}$

\begin{abstract}
Background: Gene fusions arising from chromosomal translocations have been implicated in cancer. However, the role of gene fusions in BRCA1-related breast cancers is not well understood. Mutations in BRCA1 are associated with an increased risk for breast cancer (up to $80 \%$ lifetime risk) and ovarian cancer (up to 50\%). We sought to identify putative gene fusions in the transcriptomes of these cancers using high-throughput RNA sequencing (RNA-Seq).

Methods: We used Illumina sequencing technology to sequence the transcriptomes of five BRCA1-mutated breast cancer cell lines, three BRCA1-mutated primary tumors, two secretory breast cancer primary tumors and one nontumorigenic breast epithelial cell line. Using a bioinformatics approach, our initial attempt at discovering putative gene fusions relied on analyzing single-end reads and identifying reads that aligned across exons of two different genes. Subsequently, latter samples were sequenced with paired-end reads and at longer cycles (producing longer reads). We then refined our approach by identifying misaligned paired reads, which may flank a putative gene fusion junction.

Results: As a proof of concept, we were able to identify two previously characterized gene fusions in our samples using both single-end and paired-end approaches. In addition, we identified three novel in-frame fusions, but none were recurrent. Two of the candidates, WWC1-ADRBK2 in HCC3153 cell line and ADNP-C20orf132 in a primary tumor, were confirmed by Sanger sequencing and RT-PCR. RNA-Seq expression profiling of these two fusions showed a distinct overexpression of the $3^{\prime}$ partner genes, suggesting that its expression may be under the control of the $5^{\prime}$ partner gene's regulatory elements.

Conclusions: In this study, we used both single-end and paired-end sequencing strategies to discover gene fusions in breast cancer transcriptomes with BRCA1 mutations. We found that the use of paired-end reads is an effective tool for transcriptome profiling of gene fusions. Our findings suggest that while gene fusions are present in some BRCA1-mutated breast cancers, they are infrequent and not recurrent. However, private fusions may still be valuable as potential patient-specific biomarkers for diagnosis and treatment.
\end{abstract}

\section{Background}

Gene fusions are the result of aberrant chromosomal translocations that joins together the exons of two unrelated genes, producing a chimeric mRNA transcript and protein. Many gene fusions that contribute to oncogenesis have been described in literature [1], such as the

\footnotetext{
* Correspondence: william.foulkes@mcgill.ca

'Department of Human Genetics, McGill University, Room N5-13, Stewart Biology Building, 1205 Dr. Penfield Ave, Montreal, Quebec, H3A 1B1, Canada Full list of author information is available at the end of the article
}

well-documented $B C R-A B L$ in chronic myelogenous leukemia [2]. Recently, there has been greater interest in utilizing massively parallel RNA sequencing (RNA-Seq) data to identify gene fusions [3-5]. RNA-Seq has emerged as a powerful tool to profile the entire transcriptome at a level of detail unattainable by microarrays $[6,7]$. Here, we sought to identify putative gene fusion mRNA transcripts in BRCA1-mutated breast cancers.

Mutations in BRCA1 (and BRCA2) confer a high risk for breast cancer, with a lifetime risk of up to $80 \%[8,9]$.

\section{Biomed Central}


BRCA1 mutations also confer a medium to high risk of ovarian cancer [10]. Breast cancer is a highly heterogeneous malignancy, as demonstrated by gene expression microarray studies that proposed various molecular subtypes [11,12]. BRCA1-related breast cancers have been described to share similarities with basal epithelial (basal-like) and triple-negative phenotypes [13,14]. Basal-like breast cancers have an expression profile that is similar to that found in normal basally-positioned breast epithelial cells [11], and the majority of these are triple-negative. In other words, they do not express the genes estrogen receptor (ER), progesterone receptor (PR), and human epidermal growth factor receptor 2 (HER2/neu) [15]. Furthermore, when studied by immunohistochemistry, basal-like tumors are found to express one or more of cytokeratins 5, 14, and 17, c-KIT and epidermal growth factor receptor (EGFR) [16,17].

Recurrent gene fusions have been implicated in some forms of breast cancer, such as ETV6-NTRK3 in secretory breast ductal carcinoma [18]. The role of gene fusions in BRCA1 breast cancers, however, has not been well explored. One motivation to study them in these cancers is that BRCA1 is involved in many cellular processes as well as in repairing double-stranded DNA breaks (DSBs) mediated by homologous recombination (HR) [19]. HR is error-free and involves repairing DSBs by merging two broken ends based on sequence homology [20]. When BRCA1 is deficient in HR, evidence supporting increased activity of a second repair pathway, non-homologous end joining (NHEJ), has been shown [21]. While the role of BRCA1 in NHEJ is not well known, NHEJ is more vulnerable to errors as it involves repairing DSBs by incorporating or deleting nucleotides at the site of breakage to make the two broken ends compatible for ligation [22]. Errors resulting from NHEJ can lead to increased chromosomal aberrations, translocations, and unchecked DNA damage [23]. Furthermore, Stephens et al. [24] investigated genomic rearrangements in 24 breast cancer genomes with and without BRCA1 mutations and found genomic rearrangements to be significantly widespread. Hence, we hypothesized that deficiencies in BRCA1 would cause increased chromosomal instability in a tumor cell due to impaired DNA repair pathways and NHEJ dysfunction. The resulting chromosomal lesions may potentially lead to the creation of gene fusions that can be detected in the transcriptome.

We demonstrate the use of RNA-Seq to investigate the transcriptomes of BRCA1-mutated breast cancers for gene fusions. RNA-Seq is capable of producing single-end (SE) reads (i.e. reads sequenced from only one end of the cDNA fragment) or paired-end (PE) reads ( $i$. e. both ends of the fragment are sequenced). We utilized both strategies for the discovery of gene fusions. Our analysis illustrates the feasibility of applying RNA-Seq to characterize gene fusions at the transcriptome level.

\section{Methods}

\section{Sample collection and RNA-Seq preparation}

We studied the transcriptomes of five BRCA1-mutated breast cancer cell lines (one of which is a matched lymphocyte cell line), three $B R C A 1$-mutated primary tumors, two secretory breast ductal carcinoma primary tumors (SEC1 and SEC2) and one non-tumorigenic breast epithelial cell line (Table 1). To experimentally validate and detect for recurrence of our candidate gene fusions, we additionally obtained a cDNA panel of 57 breast cancers (19 BRCA1-mutated, 17 BRCA2-mutated, and $21 B R C A 1 / 2$-wild-type). Institutional Review Boardapproved written informed consent forms were completed by all individuals whose tumor samples were used in this study.

For the samples subjected to RNA-Seq, we isolated 5 $\mu \mathrm{g}$ of total RNA from each sample and prepared them for high-throughput sequencing following the standard mRNA protocol by Illumina, unless specified otherwise. Briefly, mRNA from each sample was purified using Sera-mag magnetic oligo(dT) beads (Thermo Scientific) [for SUM149PT, Ribominus beads (Invitrogen) was applied to deplete ribosomal RNA species], fragmented at high temperatures into random fragments, and reverse-transcribed into cDNA using Superscript II (Invitrogen).

\section{Illumina Genome Analyzer sequencing}

Each prepared cDNA library was subjected to highthroughput sequencing using the Illumina Genome Analyzer IIx platform, following the standard RNA sequencing protocol. Samples were sequenced at various sites and times and are described as follows: HCC1937 was sequenced by Illumina (50 bp SE). SUM149PT, SEC1 and SEC2 were sequenced at the Institute of Cancer Research ( $36 \mathrm{bp}, 54 \mathrm{bp}$ and $54 \mathrm{bp}$ PE, respectively). SUM1315O2, HCC3153, HCC2337, T92, T50, T160 and MCF10A were sequenced at the McGill University and Genome Quebec Innovation Centre (76 bp PE per sample). All reads were processed using ELAND (AJ Cox, unpublished) to generate FASTQ files. Due to poor base quality at the 3' end of SUM1315O2 and HCC 3153, reads in these samples were trimmed by $40 \mathrm{bp}$ from the 3 ' end to produce $36 \mathrm{bp}$ high quality PE reads. Additional read statistics are summarized in Additional file 1.

\section{RNA-Seq post-processing}

Illumina FASTQ reads were first converted to Sanger FASTQ format using the ill2sanger script (patch by D Cittaro) from MAQ (http://maq.sourceforge.net/). Reads were then aligned to the human reference genome 
Table 1 Summary of RNA-Seq samples

\begin{tabular}{llll}
\hline Sample name & Sample description & BRCA1 mutation & Sequencing strategy \\
\hline HCC1937 & Cell line & 5266dupC & SE \\
SUM149PT & Cell line & 2769delT & PE \\
SUM1315O2 & Cell line & 30_31delAG & PE \\
HCC3153 & Cell line & 815 824dup & PE \\
T92 & Primary tumor & 5266 dupC & PE \\
T50 & Primary tumor & $4327 C>T$ & PE \\
T160 & Primary tumor & $5521 \mathrm{~A}>C^{*}$ & PE \\
HCC2337 & Lymphocyte cell line & $5266 \mathrm{dupC}$ & $\mathrm{PE}$ \\
MCF10A & Non-tumorigenic breast epithelial cell line & - & $\mathrm{PE}$ \\
SEC1 & Primary tumor & - & $\mathrm{PE}$ \\
SEC2 & Primary tumor & - & \\
\hline
\end{tabular}

* See Lee et al. [58] for more details

Ten breast cancer samples (eight with known germline BRCA1 mutations and two secretory breast cancers) and one non-tumorigenic breast epithelial control was sequenced using the Illumina Genome Analyzer Ilx. Only HCC1937 was sequenced using a single-end (SE) strategy, while the rest was sequenced using paired-end (PE). The cell line HCC2337 is a lymphocytic cell line derived from the same patient as HCC1937. Known BRCA1 mutations are based on RefSeq accession NM_008294.3.

(hg18) using BWA [25]. Reads that span an exon-exon splice junction will not map to the reference since it will be separated by an intron. Thus, the remainder of the unmapped reads were aligned to a custom library of junction sequences based on known RefSeq annotation. For each splice junction and read length $x$, we joined $x$ $5 \mathrm{bp}$ (or the length of the exon if less than $x-5$ ) of sequence from the upstream exon with $x-5 \mathrm{bp}$ (or the length of the exon if less than $x-5$ ) of the downstream exon. This ensured that reads mapping to a splice junction overlapped to at least $5 \mathrm{bp}$ of either end of the junction. The results were then processed using SAMTools [26] for downstream analysis. Visualization of the mapped reads was carried out using Integrated Genome Viewer (http://www.broadinstitute.org/igv).

\section{Identification of gene fusion transcripts from RNA-Seq}

In this study, we implemented two strategies adapted from methods described by Maher et al. [3,4] to facilitate gene fusion discovery with SE reads and PE reads, respectively.

In the SE approach, gene fusions are identified by checking for reads which map across the exon-exon fusion junction between the two fused genes. To simplify the analysis, we reasoned that only the subset of reads which did not fully align to the reference genome or splice junctions would be interesting (unmapped reads), as they may harbour fusion junction spanning reads. All unmapped reads were realigned to RefSeq mRNA sequences using Blat [27]. Reads that partially aligned to a RefSeq sequence (20-70\% identity) and did not fully map to anything else were retained. Ambiguous reads that were partially aligned to more than five genes were also discarded. Based on the position of the alignment, we further filtered for reads that partially aligned within 5 bp of an exon boundary using RefSeq annotation. This is because we expected that potential reads mapping across a gene fusion junction would be aligning at the exon boundaries. The resulting set of partially aligning reads was denoted as group A. Next, the unaligned portions of these reads were extracted and again subjected to alignment against RefSeq mRNA exon sequences using Vmatch [28], for effective alignment of shorter reads $(<20 \mathrm{bp})$. Reads that fully mapped to an exon and localized within $5 \mathrm{bp}$ of $5^{\prime}$ end or $3^{\prime}$ were retained in a similar fashion as before (denote as group B). Finally, we combined the results from group A with group $B$ by finding reads that had a partial alignment in group A and a full alignment in group B, thus accounting for the entire read sequence and ultimately representing a gene fusion candidate read (GFCR). All the GFCRs were tallied and summarized. Fusion partners with greater than or equal to three unique GFCRs were considered a gene fusion candidate. Each candidate was manually examined to determine its potential biological significance and to check whether it could be a false positive. This included disqualifying reads that mapped to too many loci, which may represent a repetitive region, or gene fusion candidates which mapped to two homologs that were part of the same gene family.

For the PE approach, we searched for two features which may define a gene fusion. First, we limited our selection to reads that fully and uniquely mapped to the reference genome. Second, we reasoned that reads which map to different chromosomes may reflect an interchromosomal translocation and have the potential to form a gene fusion. Alternatively, reads that map to the same chromosome but at a larger than expected distance apart may represent an intrachromosomal translocation. However, this may be confounded by large 
introns situated between two exons that the reads map to. To account for this, we used an arbitrary minimum intrachomosomal mapping distance of $1 \mathrm{Mb}$. After identifying such read pairs, we realigned the reads to RefSeq mRNA sequences using Blat. Ensuring that each read in a pair mapped to a different gene, we summarized a list of candidate gene fusions in a similar fashion as for SE reads and generated a paired end fusion score (PEFS). The scoring scheme was defined as follows: +2 for each identified read pair, -1 for each duplicate read, and -0.5 for each mismatch in a read. We prioritized those that had a minimum of three read pairs that flanked a fusion junction and had a minimum PEFS score of 5 . For further supporting evidence of each finding, we searched for additional reads that spanned across the fusion junction, analogous to the SE approach but restricted to a search space containing only the two partner genes. Here, we generated a library of all possible splice junction sequences between the 5' fusion partner and the 3 ' fusion partner, which includes the predicted fusion junction. All remaining unmapped reads were aligned to these junction sequences using Blat.

\section{Quantification of exon expression levels}

Exon expression levels were quantified by first determining the number of reads that uniquely mapped to each exonic region using BEDTools [29]. The genomic coordinates for each exon were obtained from the UCSC Table Browser [30]. The number of reads was normalized and expressed as reads per kilobase of exon model per million mapped reads (RPKM) [31].

\section{Sanger sequencing}

Primer pairs for PCR amplification and sequencing of each coding exon were generated using Primer3 [32]. Primers (see Additional file 2 for sequences) were designed to span the predicted exons forming the fusion breakpoint and to generate a maximum product size of $300 \mathrm{bp}$, which was considered optimal for amplification, purification, and sequencing. To minimize amplification of homologous genomic sequences, primer pairs were filtered using the UCSC In Silico PCR software, and only pairs yielding a single product were used. PCR reactions were performed on the cell lines' cDNA at least twice, using the following thermocycling parameters: $95^{\circ} \mathrm{C} \times 15 \mathrm{~min},\left(95^{\circ} \mathrm{C} \times 20 \mathrm{~s}, 60^{\circ} \mathrm{C} \times 20 \mathrm{~s}, 72^{\circ} \mathrm{C}\right.$ $\times 20 \mathrm{~s}$ ) for $30 \mathrm{cycles}, 72^{\circ} \mathrm{C} \times 10 \mathrm{~min}$. PCR products were purified as recommended by the manufacturer (QIAGEN). Products were sequenced by conventional Sanger methods and compared to the reference sequence to validate the gene fusion. Sequence products were obtained from MUGQIC. Sequence chromatograms were aligned and analyzed with the Staden package and Mutation surveyor software version 3.24.

\section{RT-PCR}

Total RNA (100 ng) was reverse transcribed into singlestranded cDNAs using SuperScript III reverse transcriptase (Invitrogen) and Oligo (dT) ${ }_{12-18}$ (Invitrogen) in 20 $\mu \mathrm{l}$ reaction at $50^{\circ} \mathrm{C}$ for $50 \mathrm{~min}, 85^{\circ} \mathrm{C}$ for $5 \mathrm{~min}, 37^{\circ} \mathrm{C}$ for $20 \mathrm{~min} .2 \mu \mathrm{l}$ of cDNA was used for a subsequent $20 \mu \mathrm{l}$ PCR amplification. To detect fusion transcripts, we design the forward primer targeting the 5' partner gene and reverse primer targeting the 3' partner. Primer pairs (see Additional file 2 for sequences) for the coding exons of the fusion genes were generated using Primer3 [32]. GAPDH was amplified simultaneously. PCR was performed at an annealing temperature of $60^{\circ} \mathrm{C}$ for 26 cycles. PCR products were separated by gel electrophoresis in a 3\% agarose gel (Metaphor, MRC) and visualized by ethidium bromide staining.

\section{Data access}

Sequence data of the breast cancer cell lines (HCC1937, SUM149PT, SUM1315O2, HCC3153, HCC2337 and MCF10A) from this study has been deposited to the NCBI Sequence Read Archive (http://trace.ncbi.nlm.nih. gov/Traces/sra/sra.cgi) under accession no. SRA046769.

\section{Results}

Application of RNA-Seq to identify gene fusion transcripts Initially in this study, we relied on using SE reads to identify gene fusions in the HCC1937 cell line. The remainder of this study was subsequently superseded by the PE approach, which relies on identifying read pairs that fully map to two different genes. To prioritize candidates for further review, we implemented a scoring scheme that assigned a paired end fusion score (PEFS) based on various characteristics of the read mapping. For example, a candidate with three mapped read pairs was awarded 2 points for each pair and thus had total PEFS of 6 . However, a penalty of -1 was given for each read that had the same start coordinate as another supporting read. Such reads imply that they are duplicate reads and, although it is difficult to distinguish, may represent PCR duplicates originating from the amplification step during sequencing [33]. Hence, preference was given to candidate fusions that were supported by unique reads. Furthermore, by incorporating additional reads spanning the predicted fusion junction, the $\mathrm{PE}$ approach provides two unique pieces of evidence to support the discovery of gene fusion transcripts.

\section{Proof of concept}

To evaluate our implementation, we attempted to identify known gene fusions within our samples. We applied our SE approach on HCC1937. This cell line has been previously found to have a translocation involving exon 2 of NFIA on chromosome 1 joining with exon 5 of 
$E H F$ on chromosome 11 using massively parallel DNA sequencing [24]. We were able to successfully confirm the expression of NFIA-EHF in the transcriptome of this cell line. Several 50 bp reads were mapped across the junction between exon 2 of NFIA and exon 5 of EHF (Figure 1A).

Next, we tested the PE approach on two primary tumors (SEC1 and SEC2) with secretory breast ductal carcinoma, a rare subtype of breast cancer. These samples were tested by other molecular methods to harbour the gene fusion ETV6-NTRK3 [34]. This fusion is caused by a balanced translocation, $\mathrm{t}(12 ; 15)(\mathrm{p} 13 ; \mathrm{q} 25)$, joining exon 5 of ETV6 and exon 14 of NTRK3 and is recurrent in this cancer [18]. Furthermore, functional studies have established the fusion to have a causative role in the pathogenesis of this breast cancer subtype $[35,36]$. We successfully confirmed the gene fusion transcript in SEC2 by identifying read pairs that flanked the fusion junction as well as individual reads mapping across the fusion junction (Figure 1B). The reciprocal fusion, involving exon 13 of NTRK3 as the 5' partner gene and exon 6 of ETV6 as the 3' gene, was also identified in the same sample. On the other hand, we could not find the fusion in SEC1. Since no supporting PE reads were found, we checked to see if there were only individual reads that mapped across the ETV6-NTRK3 fusion junction. To test this, we mapped our reads to a customgenerated ETV6-NTRK3 junction sequence using Blat

A

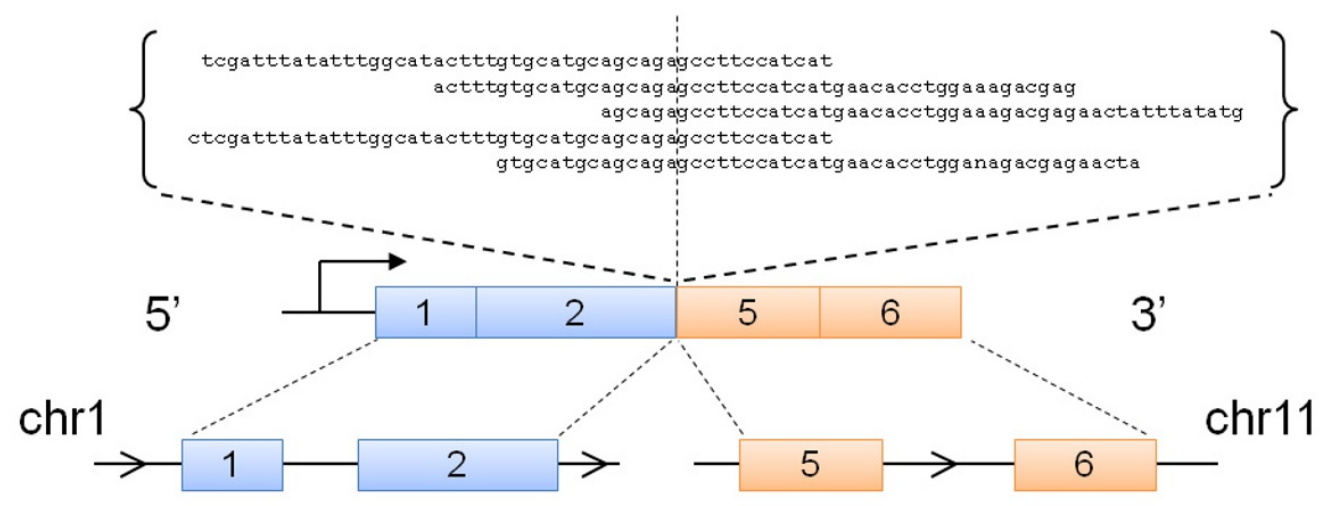

NFIA

$E H F$

B

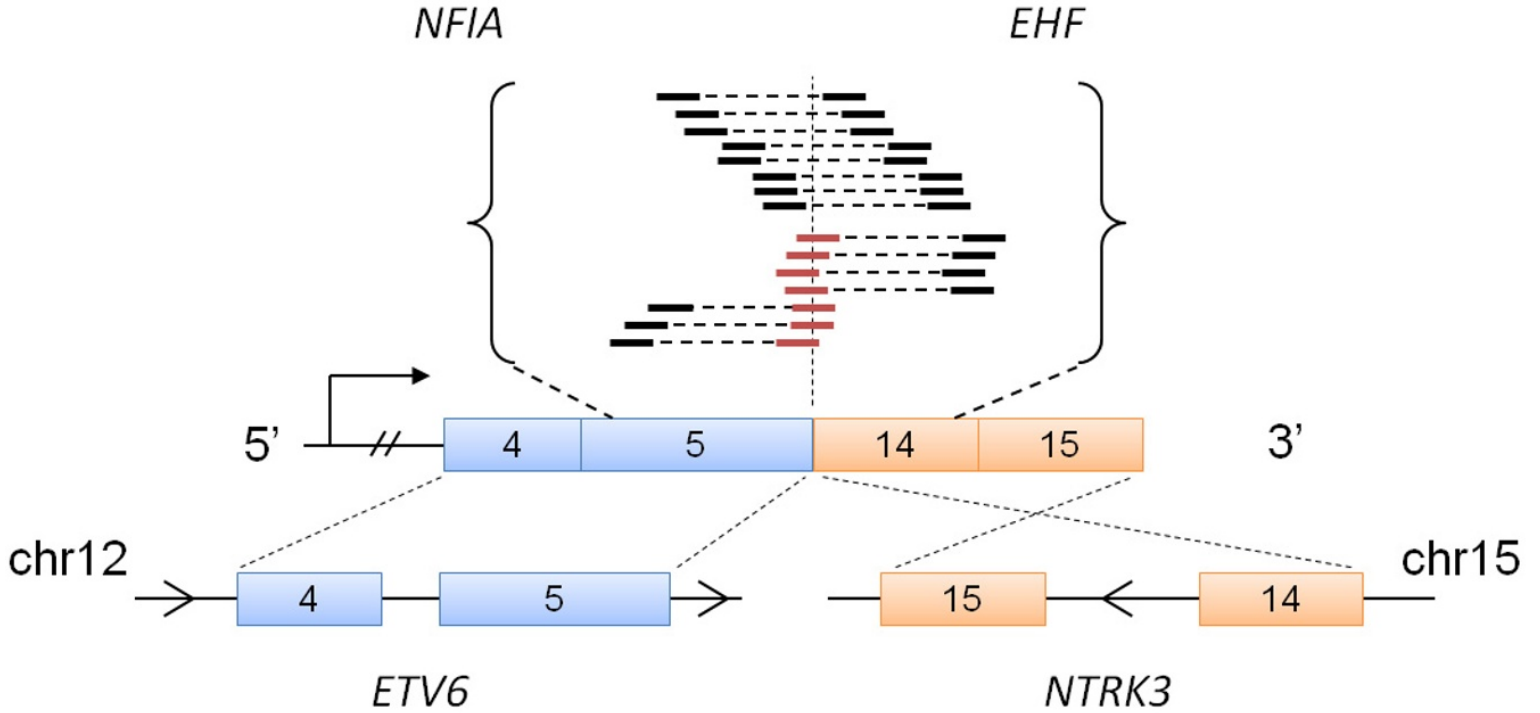

Figure 1 RNA-Seq evidence of previously described gene fusions. We first tested our SE approach on (A) the HCC1937 cell line that harbours the fusion NFIA-EHF. SE reads are shown to map across the exon-exon fusion junction between exon 2 of NFIA and exon 5 of EHF, as illustrated in the schematic. Next, we tested our PE approach on (B) two primary tumors that contain ETV6-NTRK3. Results from the sample SEC2 are shown. Paired reads (indicated by two solid lines joined by a dotted line) as well as single reads (red lines) are shown to map across the fusion junction between exon 5 of ETV6 and exon 14 of NTRK3. 
[27] and did not find any supporting reads. Subsequent inspection of starting sample from which SEC1 was prepared indicated that the tumor content was less than $50 \%$. Hence, there may have been insufficient content of this gene fusion to be detected by RNA-Seq.

\section{Discovery of gene fusions}

We analyzed the remaining samples for novel gene fusions. From the initial list of candidates, we found that many of the candidates with fewer than three supporting paired reads were due to duplicated reads, which may represent false positives. Samples T50 and T160, in particular, were sequenced at a higher depth than the other samples, yielding more sequencing data. A summary of the number of fusions found in each sample is shown in Table 2 In total, we found four candidates in samples with BRCA1 mutations that were supported by both misaligning PE reads and individual fusion junctionspanning reads. No fusions were identified in MCF10A. We manually inspected each of the four candidates and found that three of them formed in-frame transcripts, as described below and summarized in Table 3.

We identified a novel in-frame interchromosomal fusion transcript in SUM149PT involving exon 6 of $M T A P$ on chromosome 9 joining with exon 3 of $P C D H 7$ on chromosome 4 . We found five read pairs flanking the fusion junction and two individual reads spanning the junction (Additional file 3A). The 5' gene, MTAP, encodes for a methylthioadenosine phosphorylase that has been frequently observed to be co-deleted with tumor suppressor gene encoding $p 16$ in numerous cancers [37]. Interestingly, a deletion resulting in a fusion protein between MTAP and tumor suppressor gene encoding $p 15^{I N K 5 B}$ was reported in a glioma xenograft as well as other malignant cell lines [38]. The 3' gene, PCHD7, encodes for an extracellular protocadherin

Table 2 Summary of candidate gene fusions identified by paired end RNA-Seq

\begin{tabular}{llll}
\hline Sample & $\begin{array}{l}\text { PEFS } \\
>\mathbf{0}\end{array}$ & $\begin{array}{l}\boldsymbol{P E F S}>= \\
\mathbf{5}\end{array}$ & $\begin{array}{l}\boldsymbol{P E F S}>\mathbf{=} \mathbf{5} \text { plus junction spanning } \\
\text { reads }\end{array}$ \\
\hline SUM149PT & 1 & 1 & 1 \\
SUM131502 & 20 & 2 & 0 \\
HCC3153 & 8 & 1 & 1 \\
T92 & 13 & 0 & 0 \\
T50 & 1090 & 2 & 1 \\
T160 & 437 & 4 & 1 \\
HCC2337 & 14 & 2 & 0 \\
MCF10A & 10 & 0 & 0
\end{tabular}

In order to prioritize gene fusion candidates with the most read support, we selected candidates with a minimum PEFS of 5 for further investigation. A total of 12 gene fusion candidates with a PEFS $>=5$ were identified based on discordantly aligned paired reads. Of these, four candidates $(33 \%)$ were further found to have supporting reads spanning the exon-exon fusion junction.
Table 3 Candidate gene fusions supported by PE and junction spanning reads with PEFS $>=5$

\begin{tabular}{llllll}
\hline Sample & 5' $^{\prime}$ partner & $\mathbf{3}^{\prime}$ partner & PEFS & In-frame? & Reference \\
\hline HCC1937 & NFIA & EHF & N/A & Y & {$[24]$} \\
SEC2 & ETV6 & NTRK3 & 5.0 & Y & {$[18,34]$} \\
SUM149PT & MTAP & PCDH7 & 9.5 & Y & - \\
HCC3153 & WWC1 & ADRBK2 & 5.0 & Y & - \\
T50 & ADNP & C20orf132 & 10.0 & Y & - \\
T160 & CCDC126 & HSP90AA1 & 9.5 & N & -
\end{tabular}

In addition to confirming two previously described gene fusions, we identified four novel gene fusions candidates that were selected based on our filtering criteria. Among these, three of the candidates were in-frame.

protein involved in cell-cell recognition and adhesion. We were unable to amplify the MTAP-PCDH7 fusion junction using RT-PCR of cDNA in SUM149PT. One possible explanation for this failure could be due the low expression of both genes in this sample. We investigated this by examining their gene expression profiles in the RNA-Seq data, as will be discussed later.

A second in-frame interchromosomal fusion transcript was found in HCC3153 involving exon 19 of WWC1 on chromosome 5 joining with exon 10 of $A D R B K 2$ on chromosome 22 . We found three read pairs flanking the fusion junction and seven individual reads spanning the junction (Figure 2A). The 5' gene, $W W C 1$, encodes for a protein KIBRA, a cytoplasmic phosphoprotein that regulates the Hippo/SWH signaling pathway and has been shown to be involved in tumor suppression [39]. The 3' gene, $A D R B K 2$, encodes for a beta-adrenergic receptor kinase involved in the phosphorylation of $G$ proteincoupled receptors. The ATP binding and kinase domains of this gene are conserved in the predicted fusion. To verify our finding, we performed PCR amplification of the $W W C 1-A D R B K 2 \mathrm{cDNA}$ at the fusion junction followed by Sanger sequencing (Figure 2B). Furthermore, in a separate experiment not described in this study, HCC3153 was subsequently re-sequenced at the Institute of Cancer Research using RNA-Seq on the Illumina Genome Analyzer. Our analysis of this data reconfirmed the expression of the predicted fusion transcript (data not shown). To determine for recurrence, we additionally screened a cDNA panel of 57 breast cancers and did not find the fusion in any of the cases.

A third in-frame intrachromosomal fusion transcript was identified in a primary tumor T50 involving exon 2 of $A D N P$ and exon 17 of C20orf132, both of which are located on chromosome 20 (Figure 3A). Interestingly, while identifying additional reads that mapped across the fusion junction, we found read evidence supporting an additional fusion isoform of ADNP-C20orf132 involving exon 1 of $A D N P$ instead of exon 2 (Figure $3 C$ ). However, no PE reads were found to be discordantly mapped across this fusion isoform. Notably, exon 2 is 
A

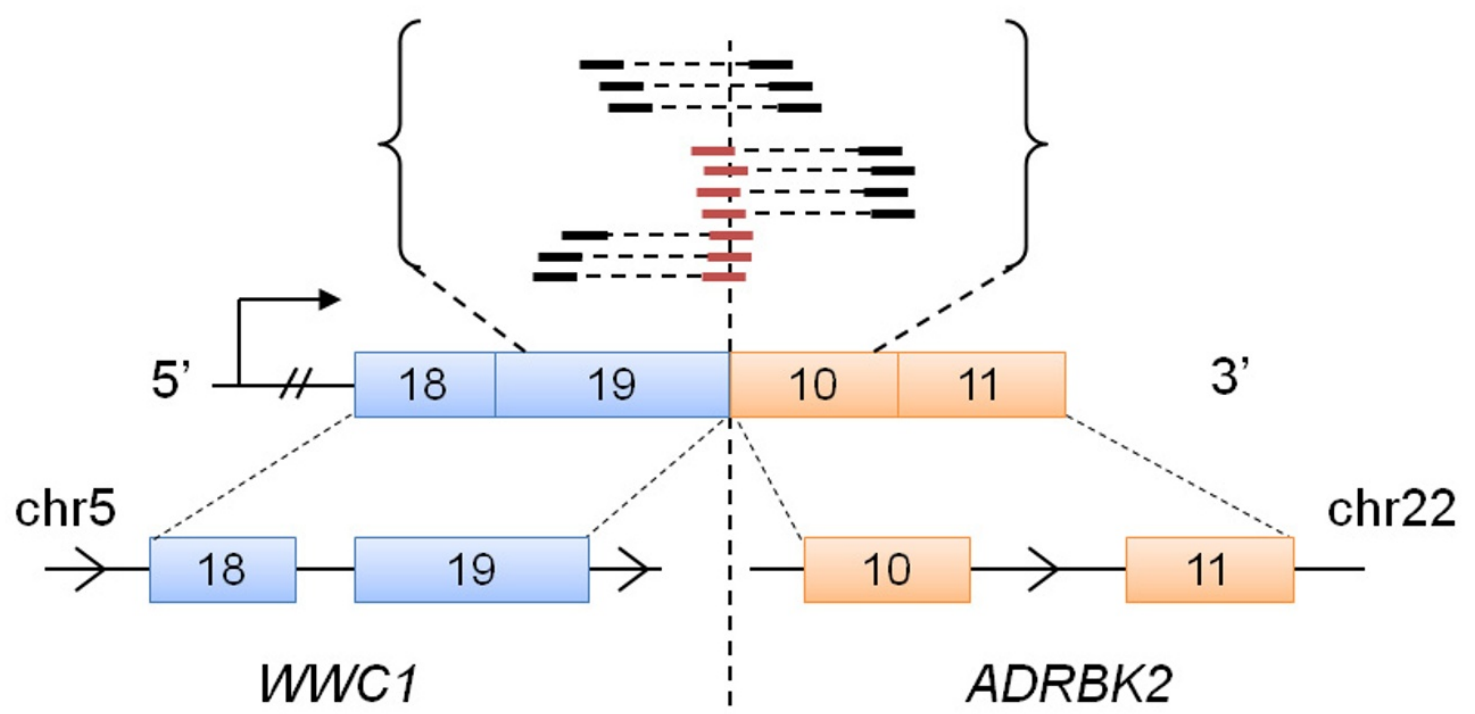

B

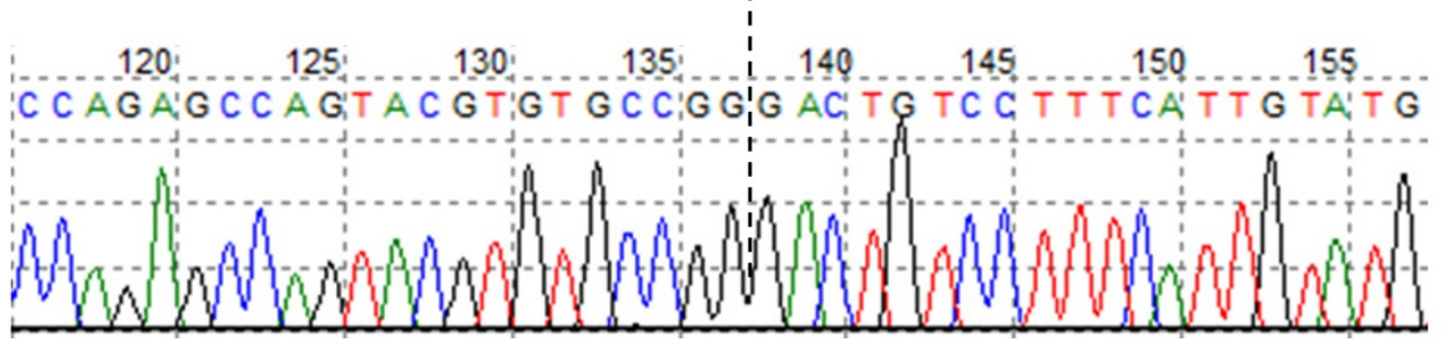

Figure 2 RNA-Seq and Sanger sequencing of WWC1-ADRBK2. We identified an in-frame gene fusion transcript in HCC1315: (A) schematic of the predicted gene fusion illustrating RNA-Seq evidence that support the fusion between exon 19 of WWC1 and exon 10 of ADRBK2. Reads are indicated by black solid lines. Paired reads are indicated by the dotted line joining two reads. Reads that span across the fusion junction are highlighted by red solid lines; and (B) the fusion junction was verified using Sanger sequencing of cDNA.

known to be alternatively spliced in $A D N P$, resulting in a long and short wild-type transcript isoforms. Both of these two exons are located in the 5' UTR of ADNP, suggesting that this gene fusion may encode for a truncated version of C20orf132. ADNP encodes for an activity-independent neuroprotector homeobox protein that has been found to be involved in cell survival, due to its proximity to a region that is frequently amplified in cancer, 20q12-13.2 [40]. On the other hand, C20orf132 is an uncharacterized protein and no functional annotation was available. We validated both fusion transcript isoforms using Sanger sequencing (Figure 3B and 3D) and RT-PCR (Additional file 4) of cDNA from the primary tumor. Furthermore, we were not able to find evidence of this gene fusion expressed in the panel of 57 breast cancer samples discussed above, suggesting that it is not recurrent.
Lastly, by sequencing HCC1937 and HCC2337, a tumor cell line and matched lymphocyte cell line derived from the same patient, respectively, we sought to determine whether NFIA-EHF was a germline mutation or an acquired somatic mutation. Our analysis could not detect the expression of this gene fusion in HCC2337, suggesting that it represents the latter. As an additional observation, we confirmed via visual inspection of the mapped reads the presence of three mutations originally reported by Tomlinson et al. [41]: 1) a 5266dupC germline mutation in BRCA1; 2) a somatic deletion of PTEN; and 3) a somatic C->T substitution in TP53 (data not shown).

\section{Expression profiling of candidate gene fusions}

We next studied the expression profiles of the candidate gene fusions based on our RNA-Seq data (Methods). 


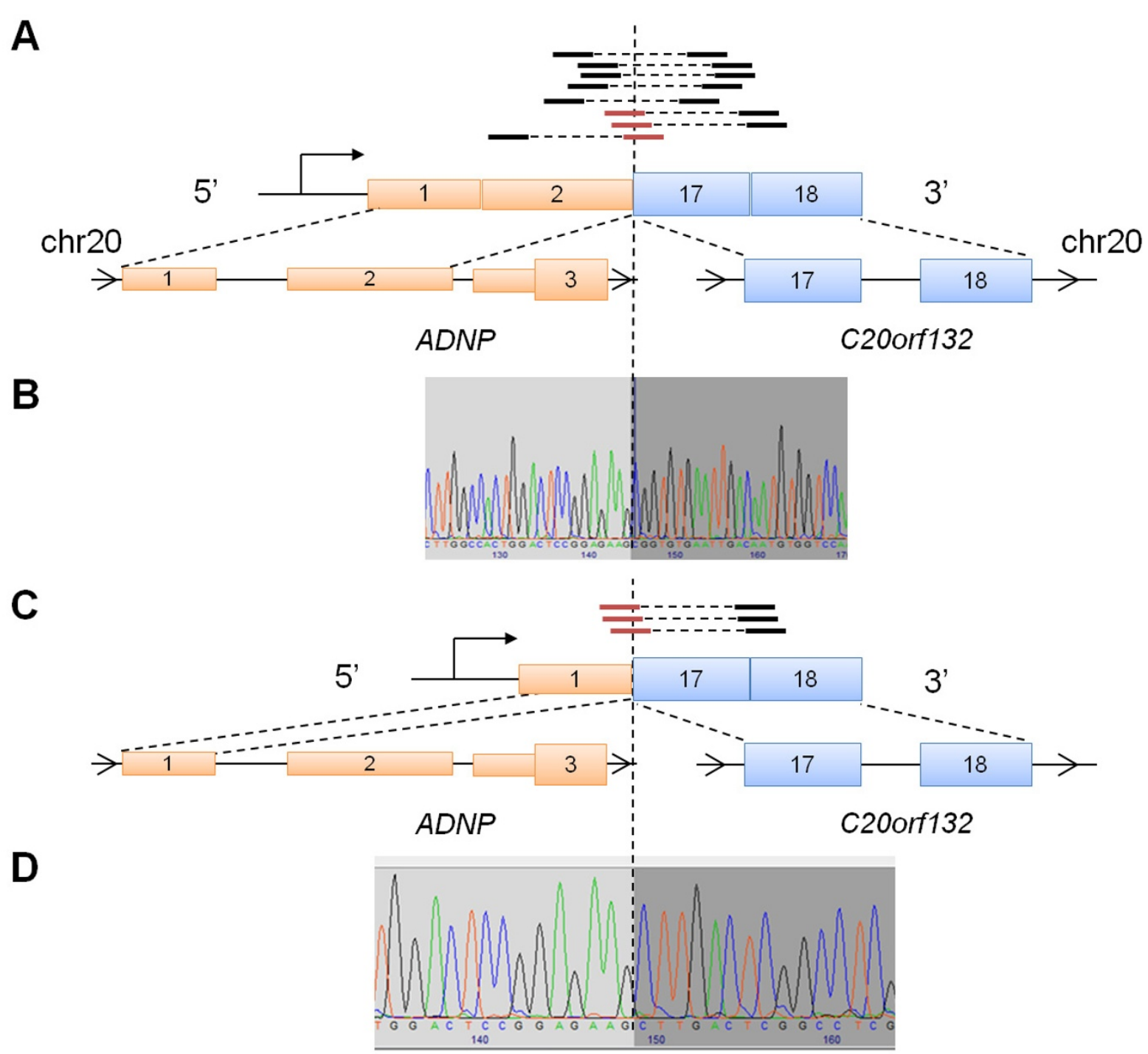

Figure 3 RNA-Seq and Sanger sequencing of ADNP-C20orf132. We identified another in-frame gene fusion in a primary tumor that was present as two transcript isoforms: (A) schematic of the first predicted gene fusion isoform illustrating RNA-Seq evidence that support the fusion between exon 2 of ADNP and exon 17 of C20orf132; (B) Sanger sequencing of the fusion junction of the first isoform; (C) while searching for fusion junction-spanning reads, we subsequently identified a second isoform in which exon 1 of ADNP was fused with exon 17 of C20orf132; and (D) Sanger sequencing of the fusion junction of the second isoform. Reads are indicated by black solid lines. Paired reads are indicated by the dotted line joining two reads. Reads that span across the fusion junction are highlighted by red solid lines.

The expression levels of every exon of each gene was quantified using a normalized metric described by Mortazavi et al. [31] called reads per kilobase of exon model per million mapped reads (RPKM). For each exon $e$, we examined the expression fold change (FC) ratio between the sample that the fusion gene is present in (denote as $e_{S}$ ) and the average of our other fusion-negative samples (denote as $\left.e_{R}\right)$. Formally, we define $\mathrm{FC}=\operatorname{RPKM}\left(e_{S}\right) /$ $\operatorname{RPKM}\left(e_{R}\right)$.

Our analysis of MTAP-PCHD7 showed that both genes were not highly expressed in SUM149PT compared to the other samples. This was illustrated by plotting the $\log _{2}$-transformed FCs of all exons in each gene, as shown in Additional file 3B. Interestingly, for WWC1-ADRBK2, we observed a discordance in expression delineated at the predicted fusion breakpoint of both partner genes (Figure 4A-B). Furthermore, the retained 3' part of $A D R B K 2$ showed increased expression over the other samples, which may be indicative of transcriptional activation driven by a foreign promoter or enhancer in $W W C 1$. To experimentally confirm that the wild-type transcripts of these two genes were not 
A

wwc1

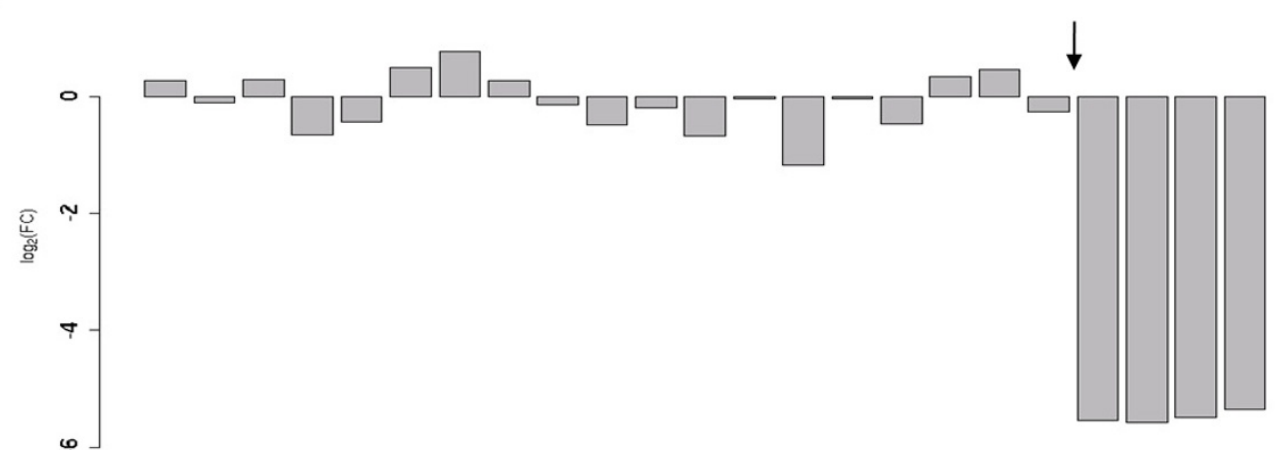

$\begin{array}{llllllllllllllllllllllllll}5 & 1 & 2 & 3 & 4 & 5 & 6 & 7 & 8 & 9 & 10 & 11 & 12 & 13 & 14 & 15 & 16 & 17 & 18 & 19 & 20 & 21 & 22 & 23 & 3 & \end{array}$

B

ADRBK2

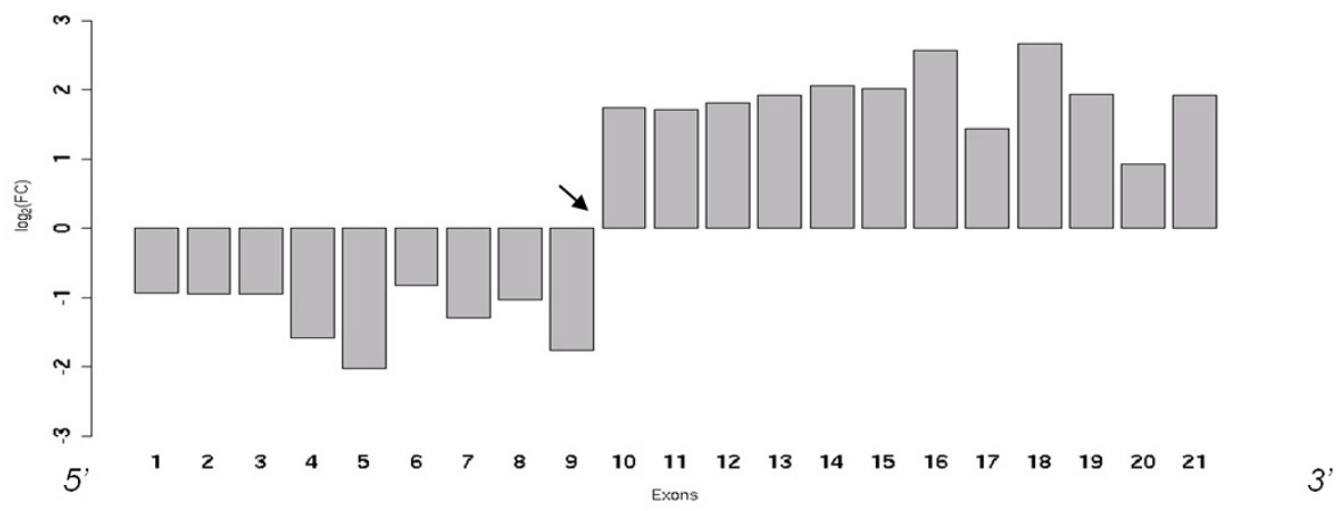

C

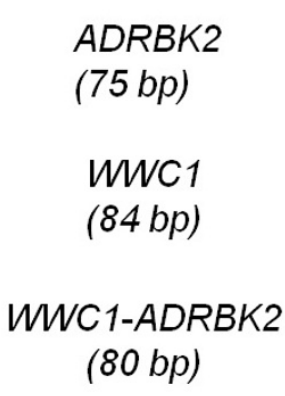

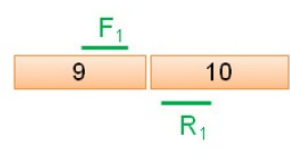
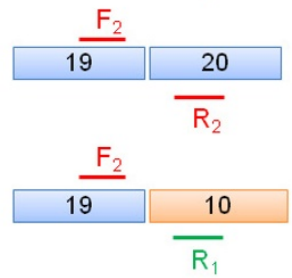

D

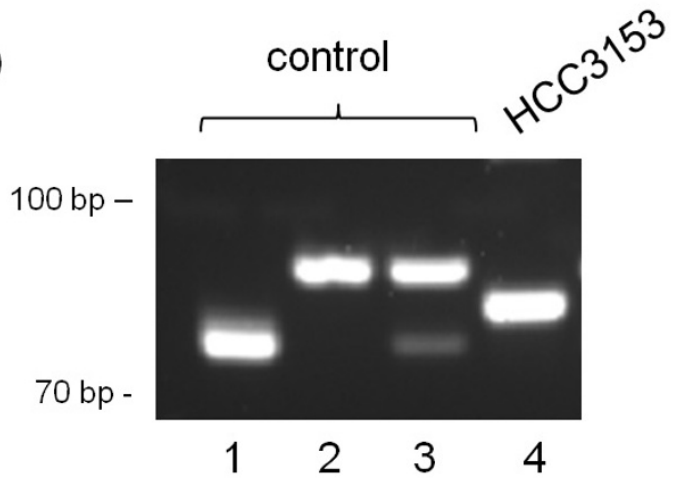

Figure 4 Expression profile of WWC1 and ADRBK2. We studied the gene expression profiles of WWC1 and ADRBK2: (A) Expression plot of WWC1 exons as measured by the $\log _{2}$ fold change (FC) between the RPKM values of each exon in HCC3153 versus the average of all other WWC1-ADRBK2-negative samples. The predicted gene fusion junction is marked by an arrow. It can be seen that exons downstream of exon 19 in WWC1 is underrepresented in HCC3153. (B) Similarly, the same can be observed in the exons upstream of exon 10 in ADRBK2. Moreover, the $3^{\prime}$ end of the gene appears to be overexpressed. (C) Two sets of primers were designed to test for expression. The first set, $F_{1}$ and $R_{1}$, spanned exons 9 and 10 of ADRBK2 to test for wild-type expression. Similarly, the second set, $F_{2}$ and $R_{2}$, spanned exons 19 and 20 of WWC1. Both sets were also combined to test for expression of predicted WWC1-ADRBK2 fusion. The expected fragment sizes are shown in brackets. (D) Exon-exon RT-PCR results: a normal control was tested for the wild-type expression of $A D R B K 2$ by $F_{1} / R_{1}$ (lane 1), the wild-type expression of $W W C 1$ by $F_{2} / R_{2}$ (2), and with both sets together (3). In lane 4, both primer sets were applied on HCC3153 and confirmed expression of the expected fusion fragment but no wild-type expression. 
expressed, we designed primers using RT-PCR (Figure 4C). As expected, only the fusion fragment was detected in HCC3153 (Figure 4D). Lastly, we repeated the exon expression analysis for ADNP-C20orf132 and similarly found discordant expression in C20orf132. Here, the exons at the 3' end of the C20orf132 (exons 17-24) were expressed higher than the 5' end (exons 1-16) (Additional file 4).

\section{Overlap with known copy number variation and chromosomal breakpoints}

Gene fusions have been shown to be linked with genomic imbalances caused by copy number variation (CNV) [42]. While we do not have the necessary genomic data to interrogate CNVs within our samples for this study, we nonetheless explored whether the genes identified above overlapped with any known structural variation in the Database of Genomic Variants [43] and previously published array comparative genomic hybridization (aCGH) data on breast cancer cell lines [44]. In the former, at least one $\mathrm{CNV}$ was identified for all six genes examined (Additional file 5A-i and 5B). Among these, $A D R B K 2$ had the most reported CNVs $(\mathrm{n}=12)$ overlapping the 5' end of the gene. The majority of these CNVs, although rare, were centred approximately 300$500 \mathrm{~kb}$ upstream of $A D R B K 2$ in a region that was enriched with segmental duplications. In the latter, the authors performed aCGH using $1 \mathrm{Mb}$ resolution arrays to study CNVs in breast cancer cell lines including SUM149PT and HCC3153. To determine whether there were copy number changes near our genes, we examined the $\log _{2}$ fold changes of BAC clone(s) whose start position was within $1 \mathrm{Mb}$ upstream and downstream of our genes (Additional file 5A-ii). For both MTAP and PCDH7 in SUM149PT, copy number losses were reported in $\mathrm{BAC}$ clones nearest these genes. In HCC3153, no BAC clones near ADRBK2 was found while copy number loss was reported near $W W C 1$. However, more studies will be required to elucidate the relationship between these genes, their predicted rearrangement, and copy number within our specific cohort. Finally, to gain insight on the frequency of chromosomal breakpoints occurring near these genes, we consulted the Mitelman Database of Chromosome Aberrations and Gene Fusions in Cancer [45] to find previously reported breakpoints occurring near these genes (Additional file 5A-iii). The chromosomal region encompassing $A D R B K 2$ was found to have the most number of cases with a reported breakpoint $(\mathrm{n}=5193)$.

\section{Discussion}

We have leveraged the power of massively parallel RNA sequencing to interrogate the transcriptomes of BRCA1mutated breast cancer cell lines and tumors for putative gene fusions. In addition to identifying previously described gene fusions, we identified three novel inframe fusions, MTAP-PCDH7 in SUM149PT, WWC1$A D R B K 2$ in HCC3153 and ADNP-C20orf132 in one primary tumor. Only the latter two were confirmed by RTPCR and Sanger sequencing.

Gene fusions can adversely affect an organism by deregulating the normal expression and disrupting the function of genes. There are two main ways in which this occurs [46]. First, the active domain of one gene is joined with a regulatory enhancer or promoter of another gene, causing an upregulation of the active domain and leading to oncogenesis. Second, a hybrid or chimeric gene fusion is formed such that characteristics from both genes are active. Interestingly, for both WWC1-ADRBK2 and ADNP-C20orf132, we observed discordant expression delineated at the predicted breakpoint region of each gene. In both cases, expression was markedly higher in the 3' partner gene compared to samples that were negative for the gene fusion of interest. Hence, this suggests that they may represent examples of the first mechanism. Gene fusions are also known to be associated with CNVs [42]. While we observed some previously reported CNVs near the selected fusion genes, no conclusions could be drawn on their functional relationship.

None of the predicted gene fusions were found to be recurrent in any of the other samples that were sequenced. This raises the question of whether these fusions represent driver mutations that directly contribute to tumorigenesis or are passenger effects that have minor or no consequence. It is well understood that driver gene fusions are typically found to be recurrent, such as BCR-ABL, ETV6-NTRK3, and TMPRSS2-ERG in prostate cancer [47], and consequently they are ideal targets for therapeutic intervention. Since we were unable to detect any of our novel fusions in our screening of additional BRCA1-mutated, BRCA2-mutated or $B R C A 1 / 2$-unrelated breast cancers, they may represent non-recurrent passenger mutations. However, more experimental studies will be required to elucidate their functional role. Moreover, many fusions that have been reported in literature have been found to be rare and have a low recurrence rate [48]. Hence, such low frequency gene fusions that are found in cancer may still be worth noting. If they can be observed in a patient, such private mutations can be potentially used as part of a personalized treatment program. For example, Leary et al. [49] recently demonstrated the ability to identify patient-specific genomic rearrangements as biomarkers in solid tumors using massively parallel sequencing. Indeed, our identification of ADNP-C20orf132 in a primary tumor represents one example of a private biomarker which may be used to track the status of the 
patient. Therefore, the use of sequencing-based approaches will be vital for advancing our understanding of tumors and to catalogue all known genetic abnormalities [50].

For this study, we focused our analysis on a collection of breast cancer samples with $B R C A 1$ mutations. Tumors of this type may possess a distinctive, possibly unique, expression signature, despite their resemblance to basallike and triple-negative breast cancers $[51,52]$. Hence, there has been great interest in elucidating the molecular mechanisms in BRCA1 cancers to identify potential biomarkers and drug targets. For example, genes that are more relied upon by tumor cells as a result of the loss of $B R C A 1$ function can be targeted for inhibition and result in cell death. This synthetic lethal relationship has led to findings of potential drug targets that are sensitive to inhibition in BRCA1 tumors, such as mitogen-activated protein kinase [53] and poly (ADP-ribose) polymerase (PARP) [54]. In the case of the latter, a PARP inhibitor, olaparib (AstraZeneca) has already been developed and undergone successful clinical trials [54]. Knowledge of the role of gene fusions in BRCA1 breast cancers, however, is limited. In a study by Stephens et al. [24], gene fusions were identified in breast cancer genomes, but none of them were recurrent. We hypothesized that mutations in BRCA1 may increase the frequency of chromosomal aberrations due to defects in the DNA repair and NHEJ pathways. This in turn, could result in the expression of novel gene fusions that can be observed at the transcriptome level. As discussed above, our analysis of a limited number of samples did not reveal strong evidence of gene fusions as major contributors to the development of BRCA1 breast cancers. However, this does not discount other genomic instabilities and lesions that arise from $B R C A 1$ mutations and are not detected by RNASeq. For example, studies have shown that BRCA1 has a role in centrosome function and the organization of chromosomes $[55,56]$. We expect future studies to involve analyzing a greater number of samples by massively parallel sequencing at the genomic and transcriptomic level, allowing for a more powerful and comprehensive interrogation of breast cancer.

We demonstrate the merits of using RNA-Seq to discover gene fusions. In particular, we note that our method to examine discordant expression between exons is related to a previously described approach to predict gene fusions using exon arrays [57]. However, while candidate fusion genes can be identified based on discordant exon expression, it can be difficult to determine which pair of genes is involved in the fusion. A sequencing-based approach can overcome this by additionally identifying reads that map across the exon-exon fusion junction. Initially, we explored using a strategy based on SE reads. Maher et al. [3] previously described an approach that leveraged both longer reads (> $250 \mathrm{bp}$ ) followed by short reads ( $35 \mathrm{bp}$ ) to find gene fusions. Since while initially working with HCC1937 we only had short 50 bp SE reads, a major challenge was to identify reads that partially aligned to two different genes. For example, a typical partial alignment may involve finding matching sequences less than $50 \%$ the length of the read (in this case, $<25 \mathrm{bp}$ ) that map to a gene. Given the large number of genes to be searched, it is likely that many of the shorter sequences will be matched in a non-specific manner. We mitigated this by filtering for matches that occur at or near the boundary of exons. Another approach was through the use of PE reads, which alleviated the reliance on finding junction spanning reads in the initial step [4]. Instead, we first systematically searched for paired reads that fully mapped to the genome but not at the expected distance or orientation, followed by searching for individual reads mapping across the predicted fusion junction. We also note two caveats to our approach. First, we identified candidate gene fusions based on RefSeq annotation for well-characterized content. As a result, some nonRefSeq genes may have been missed. Second, lowly expressed gene fusion transcripts are generally more difficult to detect if there is insufficient read coverage at the junction site and do not have supporting discordant PE reads. Thus, we focused on gene fusion candidates with adequate read coverage for further validation. In general, we found that the paired read property of PE reads allowed us to identify candidates with greater effectiveness than using only SE reads.

\section{Conclusions}

The emergence of massively parallel RNA sequencing has enabled the direct profiling of transcriptomes and revolutionized transcriptomics research. With this technology, we found that the use PE reads is well-suited for the discovery of gene fusions in cancer via bioinformatics approaches. Our investigation of several BRCA1mutated breast cancer cell lines and primary tumors revealed three previously uncharacterized non-recurrent gene fusions, two in cell lines and one in a primary tumor. While their functional role remains to be determined, no strong evidence was found to suggest that they are predominant in these cancers. However, their identification may serve as viable patient-specific biomarkers. Thus, the discovery and cataloguing of gene fusions will play an important role in the clinical treatment of solid tumours.

\section{Additional material}

Additional file 1: Read statistics of RNA-Seq samples. A summary of read statistics of the RNA-Seq samples used in this study. 
Additional file 2: Primer sequences for experimental validation. This document contains the primer sequences used for experimental validation of candidate gene fusions (Sanger sequencing and RT-PCR).

Additional file 3: Schematic and expression profile of MTAP-PCDH7 gene fusion. (A) Schematic illustrating paired-end reads that flank the fusion junction between exon 6 of MTAP and exon 3 of PCDH7. Reads are indicated by black solid lines. Paired reads are indicated by the dotted line joining two reads. Reads the span across the junction are highlighted by red solid lines; and (B) Expression plots of MTAP and $P C D H 7$ as measured by the $\log _{2} F C$ between the RPKM values of each exon in SUM149PT versus the average of all other MTAP-PCDH7-negative samples.

Additional file 4: Expression profile of ADNP-C20orf132 gene fusion. We extracted cDNA from primary tumor T50 and confirmed the presence of both isoforms of ADNP-C20orf132 by (A) RT-PCR. In both cases, we confirmed the presence of both isoforms (lanes 1 and 2) of this gene fusion. Lane 3 is a $50 \mathrm{bp}$ ladder control. (B) Expression plots of ADNP and C20orf132 as measured by the $\log _{2}$ FC between the RPKM values of each exon in the T50 versus the average of all other ADNP-C20orf132-negative samples.

Additional file 5: Genomic features overlapping or near candidate fusion genes. (A) We queried existing data to summarize known structural variation that overlaps or is within proximity of our candidate fusion genes; and (B) a list of overlapping CNVs reported in the Database of Genomic Variants.

\section{Acknowledgements}

This work was supported by funding from the United States Department of Defence (grant no. W81XWH-08-1-0402), Susan G. Komen for the Cure, Montreal Jewish Hospital Weekend to End Women's Cancer, Canadian Institutes of Health Research and Genome Canada/Génome Québec. JM is a Canada Research Chair recipient. WDF is supported by the Turner Family Cancer Research Fund and holds a Fonds de la Recherche en Santé du Québec (FRSQ) national scientist award. We would like to thank Archana Srivastava and the Génome Québec Sequencing Platform team for their assistance.

\section{Author details}

'Department of Human Genetics, McGill University, Room N5-13, Stewart Biology Building, 1205 Dr. Penfield Ave, Montreal, Quebec, H3A 1B1, Canada. ${ }^{2}$ McGill University and Genome Quebec Innovation Centre, 740 Dr. Penfield Ave, Montreal, Quebec, H3A 1A4, Canada. ${ }^{3}$ Program in Cancer Genetics, McGill University, 546 Pine Ave, Montreal, Quebec, H2W 156, Canada. ${ }^{4}$ Segal Cancer Centre, Lady Davis Institute, Jewish General Hospital, 3755 Côte-SteCatherine Road, Montreal, Quebec, H3T 1E2, Canada. ${ }^{5}$ The Breakthrough Breast Cancer Research Centre, The Institute of Cancer Research, 237 Fulham Road, London, SW3 6JB, UK. ${ }^{6}$ Institut Curie, 26 Rue d'Ulm, 75248, Paris, France. ${ }^{7}$ Department of Oncology, Lady Davis Institute, Jewish General Hospital, McGill University, 3755 Côte-Ste-Catherine Road, Montreal, H3T 1E2, Canada. ${ }^{8}$ Department of Surgery, Lady Davis Institute, Jewish General Hospital, McGill University, 3755 Côte-Ste-Catherine Road, Montreal, Quebec, H3T 1E2, Canada.

\section{Authors' contributions}

$\mathrm{KCHH}$ implemented and performed the computational analysis for gene fusion discovery in RNA-Seq data. EL performed the initial alignment of reads to the reference genome and splice junctions library. $\mathrm{CM}, J \mathrm{H}, \mathrm{CJL}$ and AA contributed to early versions of sequence alignment pipelines. $L L, L C$ and MBL designed and carried out experimental validation of the candidate gene fusions. LC performed further experimental testing on additional cohorts. RN, KF, IK and CJL carried out the massively parallel RNA sequencing at the London site. AVS provided the secretory breast carcinoma samples. MB provided the BRCA1 tumor samples. WDF provided the cell line samples. WDF also provided additional BRCA1/BRCA2-related and BRCA1 wild-type samples for validation. $\mathrm{KCHH}$ wrote the manuscript with contributions by EL, LC, JM and WDF. JM and WDF are co-principal investigators of this study. All authors have read and approved the final manuscript.

\section{Competing interests}

The authors declare that they have no competing interests.

Received: 30 July 2011 Accepted: 27 October 2011

Published: 27 October 2011

\section{References}

1. Mitelman F, Johansson B, Mertens F: The impact of translocations and gene fusions on cancer causation. Nat Rev Cancer 2007, 7:233-245.

2. Shtivelman E, Lifshitz B, Gale RP, Canaani E: Fused transcript of abl and bcr genes in chronic myelogenous leukaemia. Nature 1985, 315:550-554.

3. Maher CA, Kumar-Sinha C, Cao X, Kalyana-Sundaram S, Han B, Jing X, Sam L, Barrette T, Palanisamy N, Chinnaiyan AM: Transcriptome sequencing to detect gene fusions in cancer. Nature 2009, 458:97-101.

4. Maher CA, Palanisamy N, Brenner JC, Cao X, Kalyana-Sundaram S, Luo S, Khrebtukova I, Barrette TR, Grasso C, Yu J, et al: Chimeric transcript discovery by paired-end transcriptome sequencing. Proc Natl Acad Sci USA 2009, 106:12353-12358.

5. Berger MF, Levin JZ, Vijayendran K, Sivachenko A, Adiconis X, Maguire J, Johnson LA, Robinson J, Verhaak RG, Sougnez C, et al: Integrative analysis of the melanoma transcriptome. Genome Res 2010, 20:413-427.

6. Marioni J, Mason C, Mane S, Stephens M, Gilad Y: RNA-seq: an assessment of technical reproducibility and comparison with gene expression arrays. Genome Res 2008, 18:1509-1517.

7. Wang Z, Gerstein M, Snyder M: RNA-Seq: a revolutionary tool for transcriptomics. Nat Rev Genet 2009, 10:57-63.

8. Begg CB, Haile RW, Borg A, Malone KE, Concannon P, Thomas DC Langholz B, Bernstein L, Olsen JH, Lynch CF, et al: Variation of breast cancer risk among BRCA1/2 carriers. JAMA 2008, 299:194-201.

9. Tai YC, Domchek S, Parmigiani G, Chen S: Breast cancer risk among male BRCA1 and BRCA2 mutation carriers. J Natl Cancer Inst 2007, 99:1811-1814.

10. Antoniou A, Pharoah PD, Narod S, Risch HA, Eyfjord JE, Hopper JL, Loman N, Olsson H, Johannsson O, Borg A, et al: Average risks of breast and ovarian cancer associated with BRCA1 or BRCA2 mutations detected in case Series unselected for family history: a combined analysis of 22 studies. Am J Hum Genet 2003, 72:1117-1130.

11. Perou CM, Sorlie T, Eisen MB, van de Rijn M, Jeffrey SS, Rees CA, Pollack JR, Ross DT, Johnsen $H$, Akslen $L A$, et al: Molecular portraits of human breast tumours. Nature 2000, 406:747-752.

12. Sørlie T, Perou CM, Tibshirani R, Aas T, Geisler $\mathrm{S}$, Johnsen $H$, Hastie $T$, Eisen MB, van de Rijn M, Jeffrey SS, et al: Gene expression patterns of breast carcinomas distinguish tumor subclasses with clinical implications. Proc Natl Acad Sci USA 2001, 98:10869-10874.

13. Rakha EA, Reis-Filho JS, Ellis IO: Basal-like breast cancer: a critical review. J Clin Oncol 2008, 26:2568-2581.

14. Reis-Filho JS, Tutt AN: Triple negative tumours: a critical review. Histopathology 2008, 52:108-118.

15. Foulkes WD, Smith IE, Reis-Filho JS: Triple-negative breast cancer. N Engl J Med 2010, 363:1938-1948.

16. Arnes JB, Begin LR, Stefansson I, Brunet JS, Nielsen TO, Foulkes WD, Akslen LA: Expression of epidermal growth factor receptor in relation to BRCA1 status, basal-like markers and prognosis in breast cancer. J Clin Pathol 2009, 62:139-146.

17. Nielsen TO, Hsu FD, Jensen K, Cheang M, Karaca G, Hu Z, HernandezBoussard T, Livasy C, Cowan D, Dressler $L$, et al: Immunohistochemical and clinical characterization of the basal-like subtype of invasive breast carcinoma. Clin Cancer Res 2004, 10:5367-5374.

18. Tognon C, Knezevich SR, Huntsman D, Roskelley CD, Melnyk N, Mathers JA, Becker L, Carneiro F, MacPherson N, Horsman D, et al: Expression of the ETV6-NTRK3 gene fusion as a primary event in human secretory breast carcinoma. Cancer Cell 2002, 2:367-376.

19. Moynahan ME, Chiu JW, Koller BH, Jasin M: Brca1 controls homologydirected DNA repair. Mol Cell 1999, 4:511-518

20. Bollag RJ, Waldman AS, Liskay RM: Homologous recombination in mammalian cells. Annu Rev Genet 1989, 23:199-225.

21. Snouwaert JN, Gowen LC, Latour AM, Mohn AR, Xiao A, DiBiase L, Koller BH: BRCA1 deficient embryonic stem cells display a decreased homologous 
recombination frequency and an increased frequency of nonhomologous recombination that is corrected by expression of a brca1 transgene. Oncogene 1999, 18:7900-7907.

22. Lieber MR, Ma Y, Pannicke U, Schwarz K: Mechanism and regulation of human non-homologous DNA end-joining. Nat Rev Mol Cell Biol 2003, 4:712-720

23. Mills KD, Ferguson DO, Alt FW: The role of DNA breaks in genomic instability and tumorigenesis. Immunol Rev 2003, 194:77-95.

24. Stephens PJ, MCBride DJ, Lin ML, Varela I, Pleasance ED, Simpson JT, Stebbings LA, Leroy C, Edkins S, Mudie $L$, et al: Complex landscapes of somatic rearrangement in human breast cancer genomes. Nature 2009, 462:1005-1010

25. Li H, Durbin R: Fast and accurate short read alignment with BurrowsWheeler transform. Bioinformatics 2009, 25:1754-1760.

26. Li H, Handsaker B, Wysoker A, Fennell T, Ruan J, Homer N, Marth G, Abecasis G, Durbin R: The Sequence Alignment/Map format and SAMtools. Bioinformatics 2009, 25:2078-2079.

27. Kent WJ: BLAT-the BLAST-like alignment tool. Genome Res 2002 12:656-664.

28. Abouelhoda MI, Kurtz S, Ohlebusch E: Replacing suffix trees with enhanced suffix arrays. Journal of Discrete Algorithms 2004, 53-86.

29. Quinlan AR, Hall IM: BEDTools: a flexible suite of utilities for comparing genomic features. Bioinformatics 2010, 26:841-842.

30. Karolchik D, Hinrichs AS, Furey TS, Roskin KM, Sugnet CW, Haussler D, Kent WJ: The UCSC Table Browser data retrieval tool. Nucleic Acids Res 2004, 32:D493-496

31. Mortazavi A, Williams B, McCue K, Schaeffer L, Wold B: Mapping and quantifying mammalian transcriptomes by RNA-Seq. Nat Methods 2008, 5:621-628

32. Rozen S, Skaletsky H: Primer3 on the WWW for general users and for biologist programmers. Methods Mol Biol 2000, 132:365-386.

33. Koboldt DC, Ding L, Mardis ER, Wilson RK: Challenges of sequencing human genomes. Brief Bioinform 2010, 11:484-498.

34. Lae M, Freneaux P, Sastre-Garau X, Chouchane O, Sigal-Zafrani B, VincentSalomon A: Secretory breast carcinomas with ETV6-NTRK3 fusion gene belong to the basal-like carcinoma spectrum. Mod Pathol 2009, 22:291-298.

35. Li Z, Tognon CE, Godinho FJ, Yasaitis L, Hock H, Herschkowitz JI, Lannon CL, Cho E, Kim SJ, Bronson RT, et al: ETV6-NTRK3 fusion oncogene initiates breast cancer from committed mammary progenitors via activation of AP1 complex. Cancer Cell 2007, 12:542-558.

36. Euhus DM, Timmons CF, Tomlinson GE: ETV6-NTRK3-Trk-ing the primary event in human secretory breast cancer. Cancer Cell 2002, 2:347-348.

37. Christopher SA, Diegelman P, Porter CW, Kruger WD: Methylthioadenosine phosphorylase, a gene frequently codeleted with p16(cdkN2a/ARF), acts as a tumor suppressor in a breast cancer cell line. Cancer Res 2002, 62:6639-6644.

38. Schmid M, Sen M, Rosenbach MD, Carrera CJ, Friedman H, Carson DA: A methylthioadenosine phosphorylase (MTAP) fusion transcript identifies a new gene on chromosome 9p21 that is frequently deleted in cancer. Oncogene 2000, 19:5747-5754

39. Genevet A, Wehr MC, Brain R, Thompson BJ, Tapon N: Kibra is a regulator of the Salvador/Warts/Hippo signaling network. Dev Cell 2010, 18:300-308.

40. Zamostiano R, Pinhasov A, Gelber E, Steingart RA, Seroussi E, Giladi E, Bassan M, Wollman Y, Eyre HJ, Mulley JC, et al: Cloning and characterization of the human activity-dependent neuroprotective protein. The Journal of biological chemistry 2001, 276:708-714.

41. Tomlinson GE, Chen TT, Stastny VA, Virmani AK, Spillman MA, Tonk V, Blum JL, Schneider NR, Shay JW, et al: Characterization of a breast cancer cell line derived from a germ-line BRCA1 mutation carrier. Cancer Res 1998, 58:3237-3242.

42. Wang XS, Prensner JR, Chen G, Cao Q, Han B, Dhanasekaran SM, Ponnala R, Cao X, Varambally S, Thomas DG, et al: An integrative approach to reveal driver gene fusions from paired-end sequencing data in cancer. Nat Biotechnol 2009, 27:1005-1011.

43. lafrate AJ, Feuk L, Rivera MN, Listewnik ML, Donahoe PK, Qi Y, Scherer SW, Lee C: Detection of large-scale variation in the human genome. Nat Genet 2004, 36:949-951.

44. Neve RM, Chin K, Fridlyand J, Yeh J, Baehner FL, Fevr T, Clark L, Bayani N, Coppe JP, Tong F, et al: A collection of breast cancer cell lines for the study of functionally distinct cancer subtypes. Cancer Cell 2006, 10:515-527.

45. Mitelman Database of Chromosome Aberrations and Gene Fusions in Cancer. [http://cgap.nci.nih.gov/Chromosomes/Mitelman].

46. Rabbitts TH: Chromosomal translocations in human cancer. Nature 1994 372:143-149

47. Tomlins SA, Rhodes DR, Perner S, Dhanasekaran SM, Mehra R, Sun XW Varambally S, Cao X, Tchinda J, Kuefer R, et al: Recurrent fusion of TMPRSS2 and ETS transcription factor genes in prostate cancer. Science 2005, 310:644-648

48. Mitelman F, Mertens F, Johansson B: Prevalence estimates of recurrent balanced cytogenetic aberrations and gene fusions in unselected patients with neoplastic disorders. Genes Chromosomes Cancer 2005, 43:350-366.

49. Leary RJ, Kinde I, Diehl F, Schmidt K, Clouser C, Duncan C, Antipova A Lee C, McKernan K, De La Vega FM, et al: Development of personalized tumor biomarkers using massively parallel sequencing. Sci Transl Med 2010, 2:20ra14.

50. Velculescu VE: Defining the blueprint of the cancer genome. Carcinogenesis 2008, 29:1087-1091.

51. Chappuis PO, Nethercot V, Foulkes WD: Clinico-pathological characteristics of BRCA1- and BRCA2-related breast cancer. Semin Surg Oncol 2000, 18:287-295.

52. Foulkes WD, Stefansson IM, Chappuis PO, Begin LR, Goffin JR, Wong N, Trudel M, Akslen LA: Germline BRCA1 mutations and a basal epithelia phenotype in breast cancer. J Natl Cancer Inst 2003, 95:1482-1485.

53. Mirzoeva OK, Das D, Heiser LM, Bhattacharya S, Siwak D, Gendelman R, Bayani N, Wang NJ, Neve RM, Guan Y, et al: Basal subtype and MAPK/ERK kinase (MEK)-phosphoinositide 3-kinase feedback signaling determine susceptibility of breast cancer cells to MEK inhibition. Cancer Res 2009, 69:565-572.

54. Rehman FL, Lord CJ, Ashworth A: Synthetic lethal approaches to breast cancer therapy. Nat Rev Clin Oncol 2010, 7:718-724.

55. Pujana MA, Han JD, Starita LM, Stevens KN, Tewari M, Ahn JS, Rennert G, Moreno V, Kirchhoff T, Gold B, et al: Network modeling links breast cancer susceptibility and centrosome dysfunction. Nat Genet 2007, 39:1338-1349.

56. Wang RH, Yu H, Deng CX: A requirement for breast-cancer-associated gene 1 (BRCA1) in the spindle checkpoint. Proc Natl Acad Sci USA 2004, 101:17108-17113

57. Lin E, Li L, Guan Y, Soriano R, Rivers CS, Mohan S, Pandita A, Tang J, Modrusan Z: Exon array profiling detects EML4-ALK fusion in breast colorectal, and non-small cell lung cancers. Mol Cancer Res 2009, 7:1466-1476

58. Lee MS, Green R, Marsillac SM, Coquelle N, Williams RS, Yeung T, Foo D, Hau DD, Hui B, Monteiro AN, Glover JN: Comprehensive analysis of missense variations in the BRCT domain of BRCA1 by structural and functional assays. Cancer Res 2010, 70:4880-4890.

\section{Pre-publication history}

The pre-publication history for this paper can be accessed here: http://www.biomedcentral.com/1755-8794/4/75/prepub

doi:10.1186/1755-8794-4-75

Cite this article as: $\mathrm{Ha}$ et al: Identification of gene fusion transcripts by transcriptome sequencing in BRCA1-mutated breast cancers and cell lines. BMC Medical Genomics 2011 4:75. 